This item was submitted to Loughborough's Research Repository by the author.

Items in Figshare are protected by copyright, with all rights reserved, unless otherwise indicated.

\title{
3D-printed flat lens for microwave applications
}

PLEASE CITE THE PUBLISHED VERSION

http://dx.doi.org/10.1109/LAPC.2015.7366130

PUBLISHER

(c) IEEE

VERSION

AM (Accepted Manuscript)

PUBLISHER STATEMENT

This work is made available according to the conditions of the Creative Commons Attribution-NonCommercialNoDerivatives 4.0 International (CC BY-NC-ND 4.0) licence. Full details of this licence are available at: https://creativecommons.org/licenses/by-nc-nd/4.0/

\section{LICENCE}

CC BY-NC-ND 4.0

\section{REPOSITORY RECORD}

Zhang, Shiyu, J.C. Vardaxoglou, W.G. Whittow, and Raj Mittra. 2019. "3d-printed Flat Lens for Microwave Applications". figshare. https://hdl.handle.net/2134/20447. 


\section{D-Printed Flat Lens for Microwave Applications}

\author{
Shiyu Zhang", Yiannis Vardaxoglou", Will Whittow ${ }^{\#}$ \\ ${ }^{\#}$ School of Electronic, Electrical and Systems Engineering, \\ Loughborough University, Leicestershire, UK \\ s.zhang@lboro.ac.uk, j.c.vardaxoglou@lboro.ac.uk, \\ w.g.whittow@lboro.ac.uk
}

\author{
Raj Mittra* \\ *EMC Laboratory, University of Central Florida, USA \\ \& EE Department, KAU, Saudi Arabia \\ rajmittra@ieee.org
}

\begin{abstract}
This paper presents the design of a 3D-printed flat graded-index lens based on ray optics. The lens is comprised of several concentric dielectric rings with bespoke relative permittivities for transforming spherical waves into plane waves. 3D-printing was used to fabricate this lens with graded and tailored dielectric properties in a single process. The 3D-printed flat lens is low-cost and light-weight, but provides broadband and high gain performance. Measurement results show that the realised gain of the lens is 8 to $10 \mathrm{~dB}$ over the frequency band ranging from 12 to $18 \mathrm{GHz}$.
\end{abstract}

Keywords-ray optics, flat lens, graded index lens, 3D-printing, additive manufacturing.

\section{INTRODUCTION}

In recent years, a variety flat lens antennas have been designed based on field transformation, transformation optics, ray optics approaches and transmit-array [1]-[7]. The lenses have been designed to transform plane wave fronts into spherical wave fronts and vice versa. Antennas benefit from the lenses by realising a wide angle scanning, high gain, and formed beam.

The lenses are generally designed with radially varying reflective indices to manipulate the propagation of electromagnetic (EM) waves. For practical fabrication, the lenses often comprise several different materials with bespoke EM proprieties. However, these materials may not be available in commercial off-the-shelf (COTS) form, which means it is difficult to source ready-made materials that are available for sale to the general market. Moreover, it is challenging to vary the dielectric properties with location. Therefore, material fabrication is one of the major challenges for lens antenna applications. A number of methodologies have been investigated for synthesising materials with tailored EM properties for use in lenses [8]-[12]. The main approach of fabricating these lenses has been based on metamaterials, which suffer from narrow bandwidth, losses and dispersion [13]. Moreover the fabrication processes of the metamaterials based lenses are cumbersome at best.

This paper presents a novel wideband flat graded-index (GRIN) lens with a practical fabrication approach using 3Dprinting. The 3D-printing is an additive manufacturing technique which creates 3D-objects in successive layers. In this work, a fused deposition modelling (FDM) Makerbot ${ }^{\circledR}$ Replicator $^{\mathrm{TM}} 2 \mathrm{X}$ 3D-printer was used to fabricate the flat lens. Thermoplastic Polylactic acid (PLA) was used as the print material. The heated printer nozzle extruded the PLA material and created the lens layer by layer from the bottom up. This process enabled the fabrication of embedded micron-scale particles such as air voids in a single process without machining. Our previous research has demonstrated the viability of this approach for rapidly fabricating dielectric materials with different relative permittivities $\boldsymbol{\varepsilon}_{\boldsymbol{r}}$ by using 3Dprinting [14]. By introducing air voids into the host materials, the effective permittivity $\boldsymbol{\varepsilon}_{\boldsymbol{r} \text { eff }}$ of the mixture was determined by the volume fraction of the host material relative to air. The lens geometry was designed by using CAD tools for locally changing the infill percentage to tailor the permittivities accordingly. The entire GRIN lens was then fabricated with varying $\varepsilon_{\boldsymbol{r}}$ in one-step process using the 3D-printing. This is low-cost and enables fast automated repeatable design and manufacture.

\section{LENS DESIGN}

The flat GRIN lens was in the form of a disk and its focal point was located at its axis of symmetry. For focusing, the $\varepsilon_{r}$ of the lens material was maximum at the centre of the lens and minimum at the outermost ring. The $\varepsilon_{r}$ of the 3D-printed material PLA was characterised to be 2.72 by using NicolsonRoss and Weir method [15]. Therefore the lens was designed with $\varepsilon_{\text {reff }}=2.72$ at the centre and $\varepsilon_{\text {reff }}=1.3$ at the edge. The designed parameters are shown in Table 1.

The fabricated lens was comprised of six $10 \mathrm{~mm}$ wide discrete dielectric concentric rings. The outermost ring had the lowest effective permittivity of 1.3 , while the centre ring had the highest effective permittivity of 2.72 . The design principle of the discrete rings is shown in Fig. 3. The corresponding $\varepsilon_{\text {reff }}$ value for each ring was designed to enable them have the same focal point $O$. Hence, the lens would convert the spherical wave, emanating from the focal point into a plane wave. Fig. 2 shows the variation $\varepsilon_{\text {reff }}$ versus radial distance along the lens. In order to obtain the bespoke $\varepsilon_{\text {reff }}$. The air volume fraction was increased from the centre to the outermost with the decreased PLA infills to realising the desired effective permittivity values. Reducing the width of the rings for a smoother permittivity variation would improve the accuracy of the focal point and the gain of the lens. However, narrow rings with small variations of volume fractions were difficult to fabricate accurately due to the resolution of this printer. 
TABLE 1 DESIGNED PARAMETERS OF 3D-PRINTED LENS

\begin{tabular}{|c|c|}
\hline Parameter & Value \\
\hline Diameter & $D=120.0 \mathrm{~mm}$ \\
\hline Focus length & $F=150.0 \mathrm{~mm}$ \\
\hline Thickness & $T=18.5 \mathrm{~mm}$ \\
\hline Maximum permittivity and loss & $\varepsilon_{\text {reff }}=2.72, \tan \delta=0.008$ \\
\hline Minimum permittivity and loss & $\varepsilon_{\text {reff }}=1.30, \tan \delta=0.003$ \\
\hline
\end{tabular}

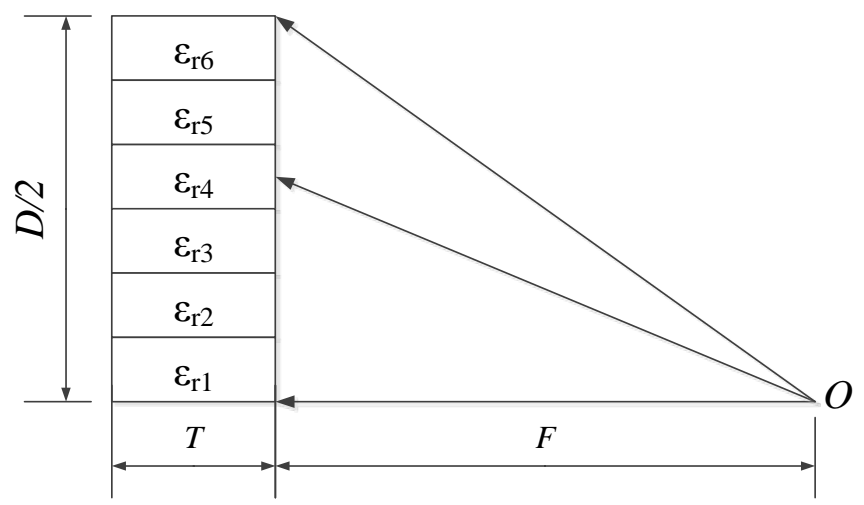

Fig. 1 Sketch of lens design principle

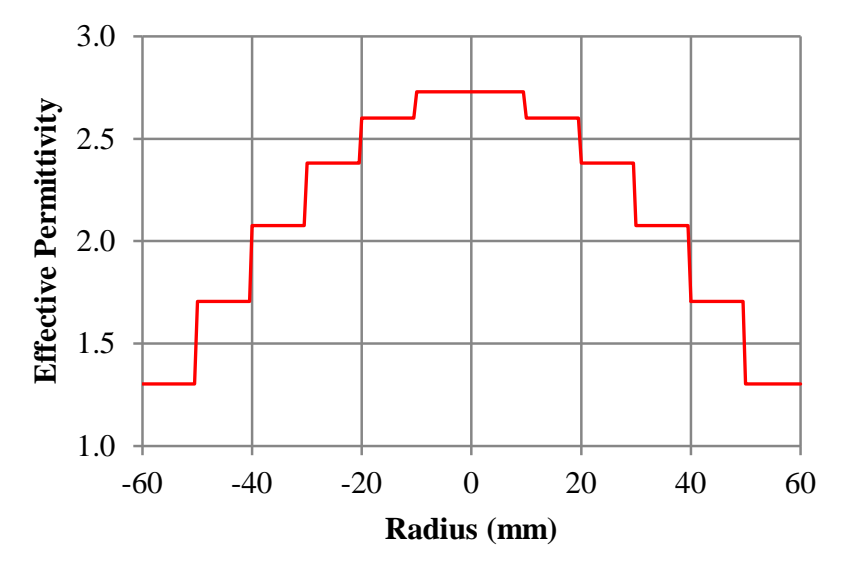

Fig. 2 Effective permittivity values for 3D-printed lens as a function of radius position

The 3D-printed flat lens with six different infill percentages in the concentric rings is shown in Fig. 1. The maximum infill at the centre was $100 \%$ and the minimum infill of the outermost ring was $10.1 \%$. The 3D-printed lens had good stiffness to resist deformation or deflection.

\section{LENS MEASUREMENT}

A boresight measurement was set up for preliminary testing of the performance of the 3D-printed lens. A Ku-band waveguide was used as the source to generate spherical wavefronts. The source was placed at the focal point of the lens axis, which was $150 \mathrm{~mm}$ away from the 3D-printed lens. A Ku-band waveguide horn antenna, which was placed at a

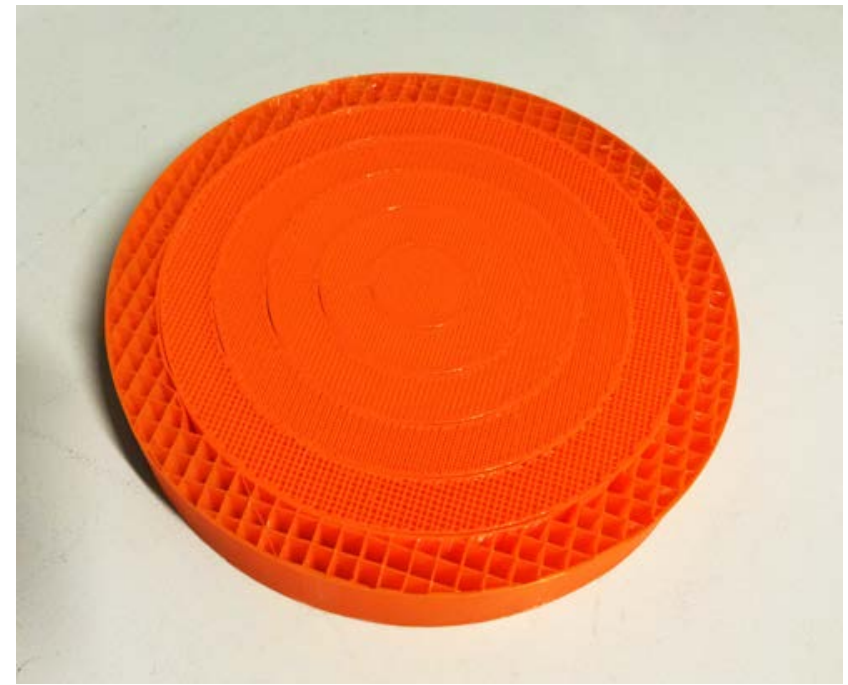

Fig. 3 3D-printed flat lens

distance of $1.5 \mathrm{~m}$ along the lens axis, served as the receiving antenna. This antenna was freely moved from theta $=-90^{\circ}$ to theta $=+90^{\circ}$ for azimuth-plane scanning.

The gain of the lens was taken as the difference between the received powers with and without the lens at boresight (theta $=0^{\circ}$ ). Fig. 4 shows the gain of the lens by normalising the received power with the lens to the power of the source at over the entire 12 to $18 \mathrm{GHz}$ range. It demonstrates the wideband performance of the lens, and that it has approximately 8 to $10 \mathrm{~dB}$ gain over the frequency of Ku-band.

The far field pattern in the $\mathrm{H}$-plane of the lens, at the centre frequency, $15 \mathrm{GHz}$, is shown in Fig. 5. The pattern of the waveguide source was also measured for as a comparison. The two patterns were normalised to the receive power at boresight of the waveguide source without the lens. The received power was increased by $8 \mathrm{~dB}$ at boresight when the lens was inserted between the source and the receiving antenna. Fig. 5 shows that the half-power beamwidth in the $\mathrm{H}$ plane is approximately $9^{\circ}$. The peak of the main beam is about $10.5 \mathrm{~dB}$ higher than the first side lobe level. The second lobes were contributed by the feed source. A larger lens or a shorter focal length would reduce the level of the side lobes.

\section{DISCUSSION}

This paper has presented a low-cost, light-weight and wideband 3D-printed flat lens which can be rapidly prototyped and be used for antenna applications. The entire lens weights approximately $130 \mathrm{~g}$ and the material cost is less than $\$ 10$. The $12 \mathrm{~cm}$ diameter GRIN lens is comprised of six concentric rings with different permittivity values. Air voids are created inside the lens during 3D-printing fabrication to reduce the permittivity of the 3D-printed material to the bespoke values. The entire lens is 3D-printed in a single process without the need for machining or assembling. The lens provides 8 to 10 $\mathrm{dB}$ gain, over the broad frequency band ranging from 12 to 18 $\mathrm{GHz}$ when illuminated by a source feed located on the axis. The directivity enhancement realised by this lens shows its potential for numerous antenna applications. 


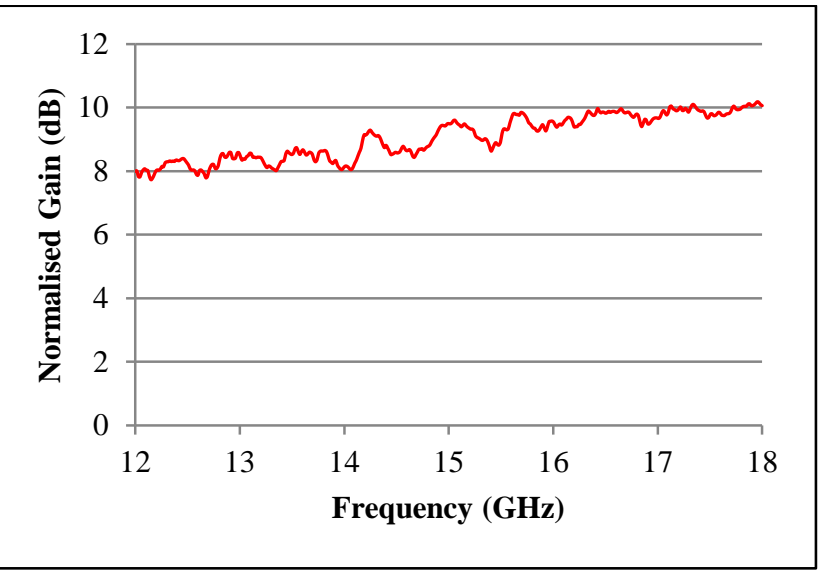

Fig. 4 Measured normalised gain of 3D-printed lens at boresight

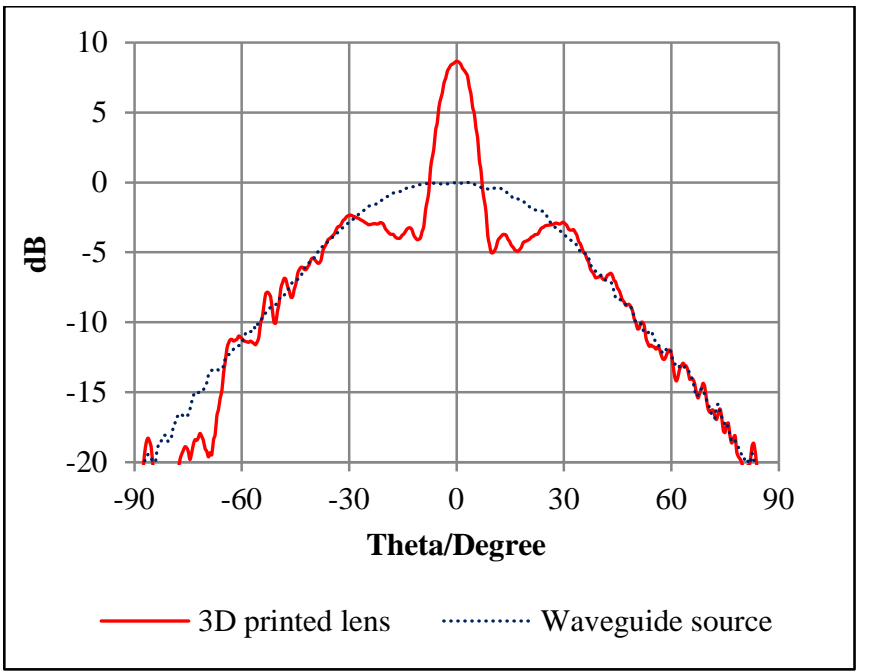

Fig. 5 Far field pattern in H-plane of lens and waveguide source at $15 \mathrm{GHz}$

\section{ACKNOWLEDGMENT}

The authors thank the colleagues Ravi Kumar Arya and Shaileshachandra Pandey for providing us the design of the lens.

\section{REFERENCE}

[1] G. Savini, P. A. R. Ade, and J. Zhang, "A new artificial material approach for flat THz frequency lenses.,” Optics express, vol. 20, no. 23, pp. 25766-25773, Nov. 2012.
[2] S. Jain, M. Abdel-Mageed, and R. Mittra, "Flat-lens design using field transformation and its comparison with those based on transformation optics and ray optics,” IEEE Antennas and Wireless Propagation Letters, vol. 12, pp. 777-780, 2013.

[3] R. Kumar Arya, S. Pandey, and R. Mittra, “A technique for designing flat lenses using artificially engineered materials,” in 2014 IEEE Antennas and Propagation Society International Symposium (APSURSI), 2014, no. iii, pp. 769-770.

[4] R. Yang, W. Tang, and Y. Hao, "A broadband zone plate lens from transformation optics.,” Optics express, vol. 19, no. 13, pp. 1234812355, 2011.

[5] Y. Zhang, R. Mittra, and W. Hong, “A zoned two-layer flat lens design,” in Final Program and Book of Abstracts - iWAT 2011: 2011 IEEE International Workshop on Antenna Technology: Small Antennas, Novel Structures and Innovative Metamaterials, 2011, pp. 412-415.

[6] O. Quevedo-Teruel, W. Tang, R. C. Mitchell-Thomas, A. Dyke, H. Dyke, L. Zhang, S. Haq, and Y. Hao, "Transformation optics for antennas: why limit the bandwidth with metamaterials?,” Scientific reports, vol. 3, p. 1903, 2013.

[7] C. G. M. Ryan, M. R. Chaharmir, J. Shaker, J. R. Bray, Y. M. M. Antar and a. Ittipiboon, "A wideband transmitarray using dual-resonant double square rings,” IEEE Transactions on Antennas and Propagation, vol. 58, no. 5, pp. 1486-1493, 2010.

[8] Y. Zhang, R. Mittra, and W. Hong, "On the Synthesis of a Flat Lens using a Wideband Low-Reflection Gradient-Index Metamaterial,” Journal of Electromagnetic Waves and Applications, vol. 25, no. 16, pp. 2178-2187, 2011.

[9] C. C. Njoku, W. G. Whittow, and J. C. Vardaxoglou, "Simulation Methodology for Synthesis of Antenna Substrates With Microscale Inclusions,” IEEE Transactions on Antennas and Propagation, vol. 60, no. 5, pp. 2194-2202, May 2012.

[10] Y. Zhang, R. Mittra, and W. Hong, "Systematic design of planar lenses using artificial dielectrics,” in 2010 IEEE International Symposium on Antennas and Propagation and CNC-USNC/URSI Radio Science Meeting - Leading the Wave, AP-S/URSI 2010, 2010.

[11] X. Chen, H. Feng Ma, X. Ying Zou, W. Xiang Jiang, and T. Jun Cui, "Three-dimensional broadband and high-directivity lens antenna made of metamaterials,” Journal of Applied Physics, vol. 110, no. 4, 2011.

[12] C. C. Njoku, W. G. Whittow, and J. C. Vardaxoglou, "Comparative study of nanomaterials' effective properties using canonical formations," in Antennas and Propagation Conference (LAPC), 2010 Loughborough, 2010.

[13] D. R. Smith, D. Schurig, M. Rosenbluth, S. Schultz, S. A. Ramakrishna, and J. B. Pendry, "Limitations on subdiffraction imaging with a negative refractive index slab,” Applied Physics Letters, vol. 82, no. 10, pp. 1506-1508, 2003.

[14] S. Zhang, C. C. Njoku, W. G. Whittow, and J. C. Vardaxoglou, "Novel 3D printed synthetic dielectric substrates," Microwave and Optical Technology Letters, vol. 57, no. 10, pp. 2344-2346, Oct. 2015.

[15] W. B. Weir, "Automatic measurement of complex dielectric constant and permeability at microwave frequencies,” Proceedings of the IEEE, vol. 62, no. 1, pp. 33-36, 1974. 